\title{
Polarization and Wavelength-Independent Time-Division Demultiplexing Based on Copolarized-Pumps FWM in an SOA
}

\author{
C. Porzi, A. Bogoni, L. Poti, Member, IEEE, and G. Contestabile
}

\begin{abstract}
A novel optical time-division demultiplexer based on copolarized-pumps four-wave mixing in semiconductor optical amplifiers is presented. The scheme is polarization and wavelength independent. Effective 10-Gb/s channel extraction from an optical time-division multiplexed $40-\mathrm{Gb} / \mathrm{s}$ aggregate frame is experimentally demonstrated.
\end{abstract}

Index Terms-Four-wave mixing (FWM), optical time-division multiplexing (OTDM), semiconductor optical amplifier (SOA), wavelength conversion.

\section{INTRODUCTION}

$\mathbf{N}$ EXT-GENERATION optical transmission systems and networks will need both very high link overall capacity and single-channel bit rate. Optical time-division-multiplexing (OTDM) and wavelength-division-multiplexing (WDM) techniques will probably cooperate to increase performances in terms of transmitted bandwidth and network granularity. Telecommunication evolution toward all-optical communications, even if with a time schedule slower than the expected, goes on through the progressive reduction of electronics. This process started with the introduction of optical amplifier and regenerators and continues toward all-optical processing.

In this frame, ultrafast OTDM systems need optical demultiplexers able to extract a tributary channel from a very high bit rate aggregate stream. To this aim, several techniques have been proposed [1]-[6], but they are generally limited by complexity or maximum operation speed. The use of semiconductor-based devices is a promising choice to implement this function because of their compactness and integrability. On the other side, they are generally speed limited with respect to fiber-based devices. However, highly detuned four-wave mixing (FWM) in semiconductor optical amplifiers (SOAs) takes advantage from ultrafast intraband phenomena like spectral hole burning and carrier heating whose characteristic response times are less than hundreds of femtoseconds. Up to now, optical demultiplexing by SOAs has been in this way demonstrated at $160 \mathrm{~Gb} / \mathrm{s}$ [6] and more, using a scheme that suffers from wavelength and polarization dependence.

Manuscript received November 10, 2004. This work was supported by the OTDM Project MURST-PROT. 9909198913-02.

C. Porzi and G. Contestabile are with Scuola Superiore Sant'Anna, Pisa 56124, Italy.

A. Bogoni and L. Poti are with CNIT, Pisa 56124, Italy (e-mail: luca.poti@cnit.it).

Digital Object Identifier 10.1109/LPT.2004.842367 (a)

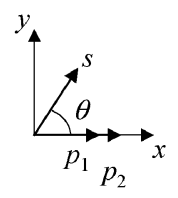

(b)

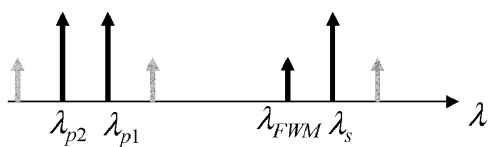

(c)

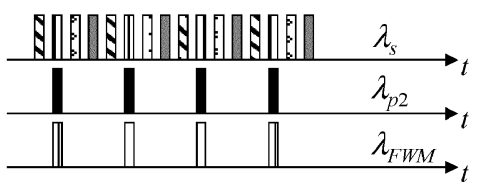

Fig. 1. Copolarized-pumps FWM scheme: (a) input states of polarization, (b) FWM spectrum, and (c) time diagram. Subscripts $p 1, p 2, s$, and FWM stand for Pump 1, Pump 2, signal, and FWM terms, respectively.

Different approaches have been proposed to overcome this polarization dependence. In [7], a clear comparison of three polarization-insensitive wavelength converters based on FWM in SOAs is presented. All those converters have proper features and can, in principle, be used in optical time demultiplexer schemes; however, up to now, only an orthogonal-pumps scheme has been experimentally demonstrated [8]. Wong and Tsang utilized orthogonal pumps and a polarization diversity scheme to obtain flat conversion efficiency together with polarization insensitive operation. In this scheme, however, one of the pumps must be properly tuned depending on data wavelength. In this letter, we use a copolarized-pumps technique to obtain polarization insensitive time demultiplexing with wavelength conversion of the extracted channel with constant conversion efficiency regardless of data wavelength. This conversion efficiency depends only by the detuning between the local pumps so that a priori knowledge of data wavelength is not required. This feature makes the demultiplexer very attractive for ultrafast hybrid OTDM/WDM systems.

\section{OPERATING PRINCIPLE}

The operating principle is schematically shown in Fig. 1 and is directly derived from [9] where the same technique is used for wavelength conversion. A wavelength converter based on copolarized pumps FWM in an SOA is polarization insensitive, although the wavelength conversion detuning and the conversion efficiency are determined by pumps detuning. These features make this scheme not properly suitable for pure wavelength conversion, but due to the wavelength transparency in respect of the incoming signal, it can be exploited to realize an optical demultiplexer for the case in which incoming data wavelength is unknown or can vary. 


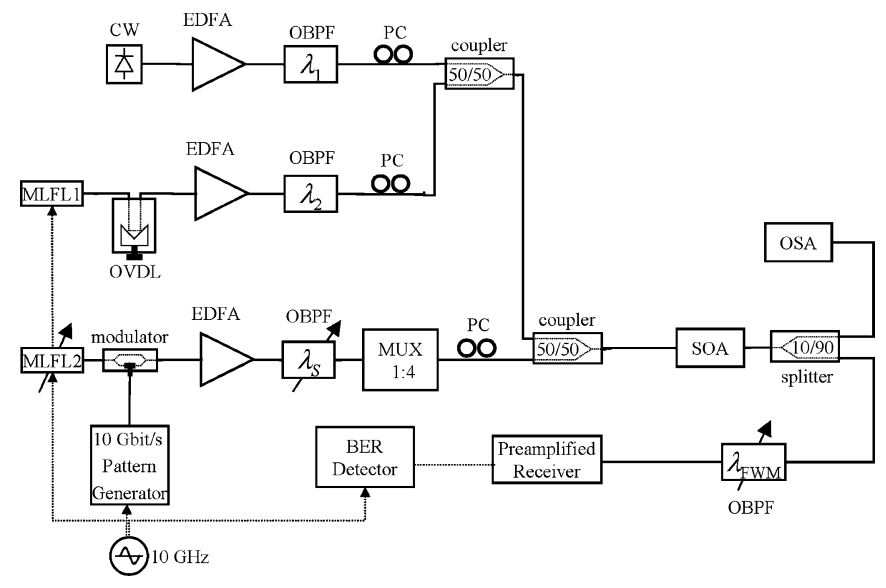

Fig. 2. Experimental setup.

In our experimental demonstration, the signal $s$ is an OTDM data stream at $40 \mathrm{~Gb} / \mathrm{s}$, Pump $1\left(p_{1}\right)$ is a continuous-wave $(\mathrm{CW})$ signal at $\lambda_{p 1}$, whereas Pump $2\left(p_{2}\right)$ is a pulsed $10-\mathrm{GHz}$ signal at $\lambda_{p 2}$. The pumps are linearly copolarized while the signal has a generic state of polarization. Here we assume, for simplicity, that it is linear, with an angle $\theta$ with respect to the pumps [see Fig. 1(a)]. The $p_{1}-p_{2}$ beating induces a gain and index modulation in the SOA. The signal $s$, while propagating through the device, experiences this nonlinear modulation producing two new side bands at the $p_{1}-p_{2}$ detuning [Fig. 1(b)].

Using the lumped model for FWM in SOAs [7], we obtain an approximated expression for the output power of the new FWM signals

$$
P_{\mathrm{FWM}}=S L_{1} L_{2} G^{3} R\left(\lambda_{p 1}-\lambda_{p 2}\right)
$$

where $S$ is the input signal power, $L_{1}$ and $L_{2}$ the pump powers, $G$ the SOA gain, and $R$ the so-called relative conversion efficiency function [10].

Equation (1) states the polarization insensitivity and, at the same time, shows that the output converted power is independent on the input signal wavelength: Output power only depends on $p_{1}-p_{2}$ detuning. Fig. 1(c) shows the time demultiplexing operation. When Pump 2 is synchronous with one of the OTDM tributary channel, we can filter out one of the new generated wavelengths to achieve demultiplexing.

\section{EXPERIMENT}

The experimental setup is shown in Fig. 2. A CW signal at $\lambda_{p 1}=1538.7 \mathrm{~nm}$ is amplified in an erbium-doped fiber amplifier (EDFA), filtered and combined with the optical system clock signal at the tributary frequency. The clock signal is generated by a mode-locked fiber laser (MLFL1) that produces $10-\mathrm{GHz}$ 6-ps-long optical solitons at $\lambda_{p 2}=1536.6 \mathrm{~nm}$. The optical clock time delay is adjusted using an optical variable delay line. An EDFA followed by an optical bandpass filter guarantees high power and low noise levels. The $40-\mathrm{Gb} / \mathrm{s}$ OTDM data signal is obtained by time multiplexing a $10-\mathrm{Gb} / \mathrm{s}$ data stream using a split and delay technique.

The $10-\mathrm{Gb} / \mathrm{s}$ tributary channel is obtained by external modulating an 8-ps optical pulse train generated by the MLFL2. The three signals are fed into the SOA with the following input

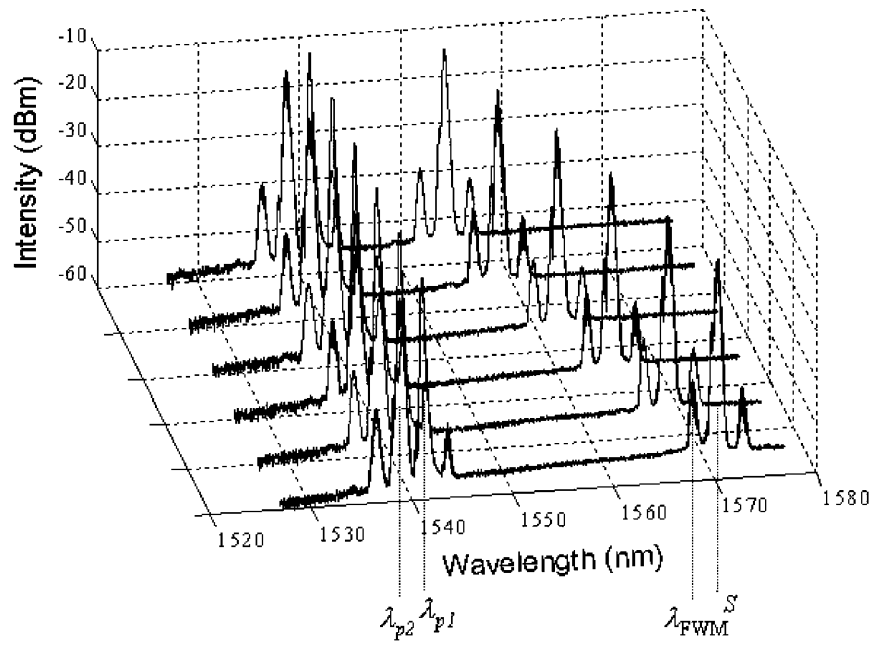

Fig. 3. Output SOA power spectrum for different signal wavelengths.

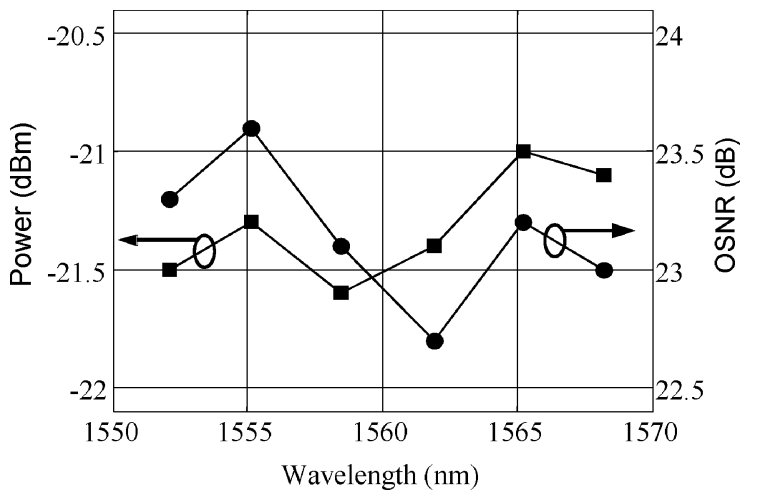

Fig. 4. Demultiplexed channel output power (left) and OSNR (right) as a function of the input channel wavelength. Maximum power and OSNR excursion: $\Delta P=0.6 \mathrm{~dB}$, and $\Delta \mathrm{OSNR}=0.9 \mathrm{~dB}$.

powers: $S=0 \mathrm{dBm}, L_{1}=7 \mathrm{dBm}$, and $L_{2}=2.5 \mathrm{dBm}$. The SOA is a polarization-independent pigtailed device with $28-\mathrm{dB}$ small signal gain and 5-dBm saturation power. The residual polarization dependence is about $0.5 \mathrm{~dB}$. The OTDM aggregate flow is wavelength tunable over the entire EDFA bandwidth due to the MLFL2 features. Since left-side FWM generated sideband (at $\lambda_{\mathrm{FWM}}$ ) has the highest conversion efficiency, thus, highest optical signal-to-noise ratio (OSNR) [11], a tunable OPBF at the SOA output selects the $\lambda_{\text {FWM }}$ signal replica. The output is finally amplified and photoreceived.

Fig. 3 shows the SOA output spectrum for six different input signal wavelengths. The two pumps have 2.1-nm fixed detuning. Moving the signal wavelength, we obtain a fixed conversion efficiency as long as the signal-pumps beatings are negligible, i.e., signal-pumps detuning is higher than $p_{1}-p_{2}$ detuning.

In Fig. 4, we show FWM term intensity as a function of the input channel wavelength together with the OSNR, calculated on 0.1-nm bandwidth.

The maximum measured output power fluctuation $\Delta P$ is limited within $0.6 \mathrm{~dB}$, whereas, the $\triangle \mathrm{OSNR}=0.9 \mathrm{~dB}$. Changing the polarization of the data signal by means of the in-line polarization controller, we measured a conversion efficiency fluctuation of less than $1.5 \mathrm{~dB}$ in the worst case at $1552 \mathrm{~nm}$. This value is due to both the SOA residual polarization dependence and to 


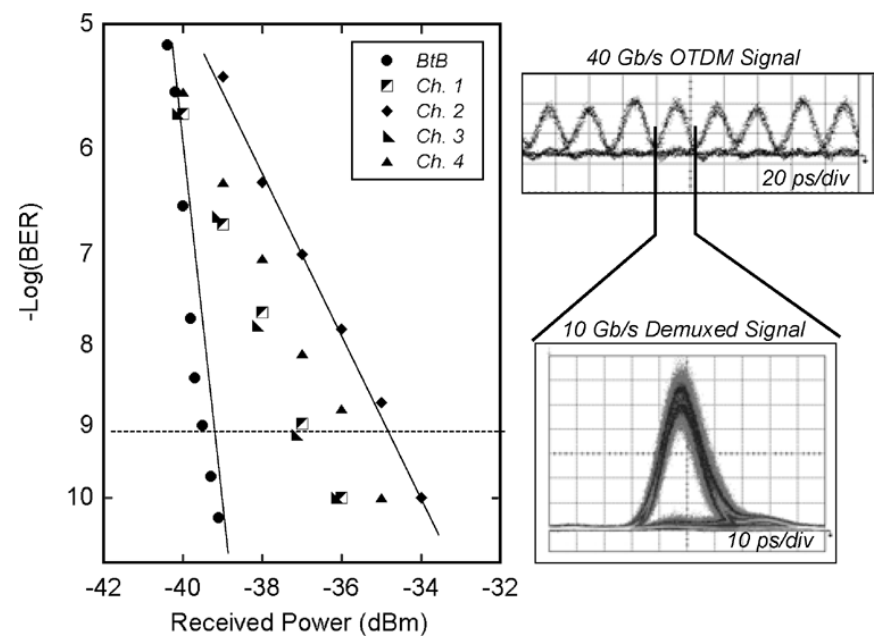

Fig. 5. BER curves of the four demuxed channels. On the right, eye diagrams of the aggregate $40-\mathrm{Gb} / \mathrm{s}$ OTDM input data stream and of one of the extracted $10-\mathrm{Gb} / \mathrm{s}$ tributary channel.

lower-efficiency FWM interaction between the two pumps and the signal.

To show effectiveness of optical demultiplexing operation of the scheme, we have performed bit-error-rate (BER) measurement for the case of lower OSNR we have found (see Fig. 4, incoming signal at $1562 \mathrm{~nm}$ ). Using an optical preamplified receiver, we obtained the curves reported in Fig. 5. With respect to the input signal in back-to-back configuration, we found no evidence of floors and a power penalty at $10^{-9} \mathrm{BER}$ in the range 2.5-4.5 dB for all the extracted channels. Intersymbol interference due to some pattern-related effect in the SOA and degradation of the OSNR contribute to power penalty. Spreading of the four channels curves is probably due to imperfect $1-4$ time multiplexing.

\section{CONCLUSION}

We have demonstrated a new copolarized pumps scheme for OTDM demultiplexing with simultaneous wavelength conversion. Using a single SOA, our technique does not depend on polarization and wavelength of the input OTDM data stream. The 40 - to $10-\mathrm{Gb} / \mathrm{s}$ demultiplexing has been experimentally demonstrated. We measured a worst case residual polarization dependence lower than $1.5 \mathrm{~dB}$ and a wavelength dependence of less than $0.6 \mathrm{~dB}$ on about $20-\mathrm{nm}$ wavelength conversion.
Power penalty for demultiplexing is less than $4.5 \mathrm{~dB}$ for a BER of $10^{-9}$.

With respect to polarization diversity and orthogonal pumps scheme, copolarized pumps permit to get free from the input signal wavelength, increasing the design flexibility, especially when such schemes are used in hybrid OTDM/WDM systems or in all-optical networks. Moreover, due to the phenomena involved in the nonlinear process, the same technique can potentially be used at $160 \mathrm{~Gb} / \mathrm{s}$ and beyond.

\section{REFERENCES}

[1] H.-F. Chou, Z. Hu, J. E. Bowers, D. J. Blumenthal, K. Nishimura, R Inohara, and M. Usami, "Simultaneous 160-Gb/s demultiplexing and clock recovery by utilizing microwave harmonic frequencies in a traveling-wave electroabsorption modulator,' IEEE Photon. Technol. Lett., vol. 16, no. 2, pp. 608-610, Feb. 2004.

[2] J. Li, B. Ollson, M. Karlsson, and P. Andrekson, "OTDM demultiplexer based an XPM-induced wavelength shifting in highly nonlinear fiber,' in Proc. OFC 2003, 2003, pp. 198-200.

[3] T. Mori, Y. Yamayoshi, and H. Kawaguchi, "All-optical time division and wavelength division demultiplexing of $160 \mathrm{Gbit} / \mathrm{s}$ OTDM signal by FWM in SOA," in Proc. LEOS 2003, vol. 1, 2003, pp. 358-359.

[4] Y. Fukuchi, T. Sakamoto, K. Taira, and K. Kikuchi, "All-optical timedivision demultiplexing of $160 \mathrm{Gbit} / \mathrm{s}$ signal using cascaded secondorder nonlinear effect in quasiphase matched $\mathrm{LiNbO}_{3}$ waveguide device," Electron. Lett., vol. 39, pp. 789-790, 2003.

[5] T. Miyazaki and F. Kubota, "Simultaneous demultiplexing and clock recovery for 160-gb/s OTDM signal using a symmetric Mach-Zehnder switch in electrooptic feedback loop," IEEE Photon. Technol. Lett., vol. 15, no. 7, pp. 1008-1010, Jul. 2003

[6] S. L. Jansen, M. Heid, S. Spalter, E. Meissner, C.-J. Weiske, A. Schopflin, D. Khoe, and H. de Waardt, "Demultiplexing $160 \mathrm{Gbit} / \mathrm{s}$ OTDM signal to $40 \mathrm{Gbit} / \mathrm{s}$ by FWM in SOA," Electron. Lett., vol. 38, pp. 978-980, 2002.

[7] J. P. R. Lacey, M. Summerfield, and S. J. Madden, "Tunability of polarization-insensitive wavelength converters based on four-wave mixing in semiconductor optical amplifiers," J. Lightw. Technol., vol. 16, no. 12 , pp. 2419-2427, Dec. 1998.

[8] C. S. Wong and H. K. Tsang, "Polarization-independent time-division demultiplexing using orthogonal-pumps four-wave mixing," IEEE Photon. Technol. Lett., vol. 15, no. 1, pp. 129-131, Jan. 2003.

[9] R. Schnabel, U. Hilbk, T. Hermes, P. Meißner, C. Helmolt, K. Magari, F Raub, W. Pieper, F. J. Westphal, L. Ludwig, L. Küller, and H. G. Weber, "Polarization insensitive frequency conversion of a 10-channel OFDM signal using four-wave-mixing in a semiconductor laser amplifier," IEEE Photon. Technol. Lett., vol. 6, no. 1, pp. 56-58, Jan. 1994.

[10] J. Zhou, N. Park, K. J. Vahala, M. A. Newkirk, and B. I. Miller, "Fourwave mixing wavelength conversion efficiency in semiconductor travelling wave amplifiers measured to $65 \mathrm{~nm}$ of wavelength shift," IEEE Photon. Technol. Lett., vol. 6, no. 8, pp. 984-987, Aug. 1994.

[11] G. Contestabile, F. Martelli, A. Mecozzi, L. Graziani, A. D’Ottavi, P. Spano, G. Guekos, R. Dall'Ara, and J. Eckner, "Efficiency flattening and equalizing of frequency up- and down-conversion using four-wave mixing in semiconductor optical amplifiers," IEEE Photon. Technol. Lett., vol. 10, no. 10, pp. 1398-1400, Oct. 1998. 